\title{
One size does not fit all: Pharmacists' perspectives on disparities in quality care provided to adult patients on the Autism Spectrum Disorder
}

\author{
Ola A. Ghoneim ${ }^{1 *}$, Heba A. Eassa ${ }^{2}$, Luis M. Lavoura ${ }^{2}$, Noelle Bianculli ${ }^{2}$ and Charlotte Mann ${ }^{3}$ \\ ${ }^{1}$ Department of Pharmaceutical and Administrative Sciences, Western New England University-College of Pharmacy and \\ Health Sciences, Springfield, MA 01119, USA \\ ${ }^{2}$ Department of Pharmaceutical Sciences, School of Pharmacy \& Physician Assistant Studies, University of Saint Joseph, \\ Hartford, CT 06103, USA \\ ${ }^{3}$ Department of Counselling and Applied Behavioral Studies, School of Interdisciplinary Health and Sciences, University \\ of Saint Joseph, West Hartford, CT 06117, USA \\ * Correspondence: e-mail: ola.ghoneim@wne.edu Ola Ghoneim, Department of Pharmaceutical and Administrative \\ Sciences, Western New England University-College of Pharmacy and Health Sciences, 1215, Wilbraham Rd, \\ Springfield, MA 01119, USA.
}

\begin{abstract}
The aim of this work is to improve the overall healthcare experience of patients with Autism Spectrum Disorder (ASD), this study was conducted to collect the perspectives of healthcare professionals' (mainly pharmacists) on current healthcare barriers for patients with ASD. The study aims to identify possible interventions to minimize the patient-provider communication gap, that takes in consideration the social skills and language/communication level of patients with ASD. The ultimate goal is improving the healthcare experience for patients on the spectrum and narrow the communication gap between adult patients with ASD and their trusted pharmacists. An online questionnaire was created using Google Forms and results were directly extrapolated into easy-to-read tables, and graphs. Questionnaire was sent to healthcare providers. All participants were kept anonymous, and de-identified. A total of 116 participants completed the online questionnaire. Most participants came from hospitals-inpatient, followed by community pharmacies, and hospitals-outpatient, and span across all years of practice. Responses from female providers were significantly higher. The majority of providers did not receive any additional training, and do not believe they are comfortable communicating and/or prepared to provide quality care to patients with ASD. Providers desire to bridge the gap and explore novel communication tools. Information from this study will be the seed to develop an innovative communication tool for transfer/exchange/retrieval of information between ASD patients and their providers. The communication aid will be piloted in the next phase of this research.
\end{abstract}

Keywords: Autism Spectrum Disorder (ASD); communication; disparities; health care; pharmacists.

This is an open access article distributed under the CC BY-NC-ND license https://creativecommons.org/licenses/by/4.0/

\section{INTRODUCTION}

Achieving health equity, eliminating health disparities, and attaining health literacy to improve the well-being of all, is one of the overarching goals of Healthy People 2020 (and its fifth iteration Healthy People 2030), a nationwide healthpromotion and disease-prevention initiatives set by the United States Department of Health and Human Services. It is exceedingly encouraging to see that two of the four objectives monitoring barriers to health care for people with disabilities exceeded their goals by 2020 . However, in the midcourse review ${ }^{1}$, two objectives had showed little or no detectable change; the proportion of youth aged 12-17 with special health care needs receiving pediatric-to-adult health care transition planning, and the use of inappropriate medications among persons aged 65 and over with disabilities. ${ }^{2}$

Our team is particularly concerned about the quality of care for patients with Autism Spectrum Disorder (ASD). ASD is a group of pervasive 
neurodevelopmental disorder that is reported in all racial, ethnic, and socioeconomic groups. The prevalence of ASD is on the rise estimated with 1 in 44 children (4 times more common among boys than girls) according to CDC ${ }^{3}$. Whether it is a true incidence of ASD is rising, or an artifact of increased surveillance, early diagnosis and awareness, the fact is there is at least 1.5 million individuals on the spectrum are currently living in the US. ${ }^{4}$ The population of adults on the spectrum is growing with about 50,000 patients on the spectrum turning 18 years old/year in the US. More than half of individuals with ASD have four or more comorbidities and the picture for prevalence and outcomes in older adults are alarming. ${ }^{5}$ This poorly understood disorder has a major impact on the lives of, not only the individuals living with the disorder, but also the lives of their families and caregivers. Projected cost to society is expected to reach $\$ 11.5$ trillion dollars annually by $2029 .{ }^{6}$

Therefore, we have been especially interested in the quality of life for individuals on ASD spectrum, and those caring for them. ${ }^{7,8} \mathrm{~A}$ major component of the quality of life of any individual is having a healthy life and receiving quality care from all health care providers with no health disparities. Logically, the first step of this effective unbiased health care plan is appropriate communication, which is by definition, the form of a transfer and exchange of information that helps both sender and recipient get a better understanding of the other in order to meet that person's needs or wants. ${ }^{9,10}$

It is well known, however, that communication difference is one of the two main domains when diagnosing an individual with ASD along with repetitive behaviors and restricted interests according to the Diagnostics Statistical and Manual $5^{\text {th }}$ edition in 2013. ${ }^{11}$ Adult individuals on ASD often face challenges communicating with others, even if they are verbal communicators. ${ }^{12-14}$ Some adults have difficulty communicating even nonverbally (i.e.) through hand gestures, eye contact, tone of voice, body language, and facial expressions. 12, 13, 15, 16

Nicolaidis et al, 2016 eloquently illustrated that although ASD is a spectrum, individuals do not fall on one linear spectrum of "low" and "high" functioning, instead, skills or challenges fall along spectra on multiple axes (spoken language, written communication, need for consistency, sensory sensitivity, emotional regulation, and so forth). An individual with no spoken language may be able to read and write at a graduate level, whereas, an individual who speaks fluently may have profound learning disabilities. Within each axis, skills can change depending on environmental stimuli, supports, and stressors. ${ }^{16}$
The puzzling described communication difference makes it extremely difficult for pharmacists, among other healthcare providers, to communicate directly and efficiently with their patients, provide consultation, appropriate drug information and stipulate patient counseling points. Providers often find themselves communicating with the caregivers instead, regardless of the age group of their patients, which is usually understandable and/or welcomed in the case of pediatric ASD patients. However, this form of health disparities is unwelcomed when the patient is an adult on the spectrum, adding another level of frustration to the patient. ${ }^{17,18}$ Although, there is an assumption that patients with ASD live with their caregivers, and the vast majority do, the level of needs vary from one individual to another, which adds to the complexity of providing quality of care.

Many adults on the spectrum lack sufficient knowledge regarding important health topics, such as healthy hygiene habits, living a healthy lifestyle with exercise/diet, preventive medical and dental visits, and medication adherence. A variety of societal, behavioral and environmental factors contribute to this lack of information. ${ }^{19,20}$ Christine Preißmann (a medical doctor who was diagnosed with ASD at age 27) highlighted some of the hurdles to almost every healthcare setting from ASD patients' point of view. This combination of being an individual on the spectrum, and with a professional medical background is of special value to this research topic. These hurdles include difficulty in scheduling appointments, choosing the medical provider, anxiety and distress due to changes in routines and sensitivity to touch among others. ${ }^{21}$

As the prevalence rate continues to rise, more and more adult individuals on the spectrum are navigating the healthcare system for their medical needs. Autism, like other disabilities, does not preclude one from being healthy. The vast majority of these individuals are on daily medications and are patients for life. Therefore, the demand for optimal quality care by health care providers is greater than ever before. ${ }^{18}$ In 2012, a systematic review of the literature to determine whether differences exist for children with ASD versus children without ASD in the utilization, accessibility, and cost of their health care service. ${ }^{22}$ The review revealed that children with ASD generally have higher health care utilization rates and costs. However, they generally have worse access to care and medical homes. ${ }^{22}$ Additionally, a cross-sectional retrospective cohort study was conducted to compare the prevalence of physical and mental health conditions in a national sample of autistic older adults to a matched population comparison cohort. ${ }^{23}$ Results were astonishing. Autistic older adults had significantly greater odds of nearly all physical health conditions 
including epilepsy, Parkinson's disease, and gastrointestinal conditions, as well as most mental health conditions, including schizophrenia and psychotic disorders, attention deficit disorders, and suicidality or self-inflicted injury. ${ }^{23}$ Raymaker et al., 2017 also confirmed that adults on the spectrum experienced difficulties with health care access at higher rates than people with other disabilities. ${ }^{18}$

Despite the increased interest by certain health care providers in working with patients on ASD, studies highlighted that there is a shortage in adequate healthcare transition services, incorrect assumptions about individual patients, use of accessible language, and openness to providing other accommodations. 24-26 Thus, there remains a considerable discrepancy between the number of individuals on ASD seeking medical care and the number of healthcare professionals willing to provide care to them, which may lead to long waiting periods, delays in diagnosis, improper treatment of medical conditions, inadequate patient counseling, and much more. ${ }^{26}$ Given the growing number of patients on ASD and the communication deficits inherent in the diagnosis that may impact health care access, we became increasingly interested in learning how health care providers are prepared to serve patients with ASD.

Our objectives in this study were:

1. Obtain healthcare providers' prospective (with special interest in the perspectives of pharmacists) on the current healthcare barriers and/or disparities for adult patients on the spectrum.

2. Gather any additional trainings/ certificates/ licenses that help healthcare providers provide quality care for ASD adult patient population.

3. Learn about specific communication protocol(s)/guidelines in places in their current practices.

4. Gauge their interests in possible ways to improve the current quality of care and the need to develop an interactive augmentative communication instrument/tool that takes in consideration the social skills and language/communication level of adult patients with ASD.

\section{METHODS}

Due to the fact that this research has been conducted as part of the School of Pharmacy Advanced Pharmacy Practice Experience, we became more interested in learning also the pharmacists' perspective on disparities in quality care provided to adult patients on ASD. Pharmacists, among all healthcare providers, are the topmost trusted health care professionals and the most accessible ones. ${ }^{27}$ They have an important responsibility as the last line of communication before the patients go home. Pharmacists are trained to provide patient counseling and recommendation and they are the drug experts. ${ }^{28}$ They have an important responsibility as the last line of communication before the patients go home.

An online questionnaire was created for healthcare providers to provide their professional perspectives on the current healthcare barriers and/or disparities for adult patients on ASD, in addition to their perspective on how prepared/equipped they believe they are to serve the population of patients on the spectrum. The questionnaire was generated on Google Forms. The readability and completion time were tested prior to questionnaire launching by faculty members of our School of Pharmacy from the Department of Pharmacy Practice, who are also practicing pharmacists.

The results were directly extrapolated into easyto-read tables, graphs, and scales. The research study and questionnaire were approved to be used by our Institutional Review Board (IRB Protocol Number: 18-0054). All participants were kept anonymous, and de-identified.

The questionnaire comprised of 18 questions including demographic questions $(n=4)$, Likert-scale items $(n=2)$, short answer questions $(n=5)$, and multiple choice questions $(\mathrm{n}=7)$ yielding both quantitative and qualitative data (questionnaire is attached under supplemental materials). A demographics section was included to collect participant information regarding their professional licensure, years of experience, type of healthcare setting. several questions recorded healthcare provider's perceived perspectives on whether they feel prepared to provide patients with ASD quality care and how comfortable they are in communicating with patients with ASD.

A short answer question was used for providers to indicate what areas in patient care they felt most confident in providing quality care. Additionally, series of questions recorded any additional degrees, licenses, or certifications the provider may have received either on their own or through their employer as well as any tools or guidelines that could be used to improve ASD patient care. Two finals questions addressed barriers to ASD patient care and potential solutions to current practice. The online link to the questionnaire and a synopsis of the research study was sent in an approved email to community pharmacies, hospitals, clinics, dental offices, and healthcare organizations in the Northeast. All participants are kept. anonymous and their responses were kept confidential, and de-identified. Table 1 illustrates the18 questions addressed and answer choices 
Ghoneim et al, Azhar Int J Pharm Med Sci 2022; Vol 2 (1):124-135

Table 1. Questionnaire questions

\begin{tabular}{|c|c|c|c|c|c|c|c|c|}
\hline Questions & \multicolumn{8}{|c|}{ Answers (choices) } \\
\hline 1. What is your gender? & Female & Male & Prefer not to say & $\begin{array}{c}\text { Other:.............. } \\
\ldots \ldots .\end{array}$ & & & & \\
\hline 2. How long have you been practicing? & less than 5 years & $5-10$ years & $10-15$ years & $15-20$ years & $\begin{array}{c}\text { greater than } 20 \\
\text { years }\end{array}$ & & & \\
\hline 3. Which of these titles do you hold? & $\begin{array}{l}\text { Nursing: LPN, } \\
\text { RN, APRN }\end{array}$ & $\begin{array}{l}\text { Dentistry: } \\
\text { DMD or } \\
\text { DDS }\end{array}$ & $\begin{array}{c}\text { Pharmacy: RPh or } \\
\text { PhamD. }\end{array}$ & $\begin{array}{c}\text { Medicine: MD } \\
\text { or DO }\end{array}$ & Other & & & \\
\hline $\begin{array}{l}\text { 4. Which of these describes your practice } \\
\text { setting? }\end{array}$ & Hospital- inpatient & $\begin{array}{l}\text { Hospital- } \\
\text { outpatient }\end{array}$ & Outpatient clinic & $\begin{array}{l}\text { Independent } \\
\text { pharmacy }\end{array}$ & $\begin{array}{c}\text { Community } \\
\text { pharmacy }\end{array}$ & Dental office & $\begin{array}{l}\text { Physician's } \\
\text { office }\end{array}$ & Other \\
\hline \multirow{2}{*}{$\begin{array}{l}\text { 5. As a provider, are you prepared to provide } \\
\text { patients with Autism Spectrum Disorder } \\
\text { (ASD) quality care? }\end{array}$} & Very Unprepared & & & & Very Prepared & & & \\
\hline & 1 & 2 & 3 & 4 & 5 & & & \\
\hline $\begin{array}{l}\text { 6. As a provider, how comfortable are you in } \\
\text { communicating with patients with ASD? }\end{array}$ & $\begin{array}{c}\text { Very } \\
\text { Uncomfortable }\end{array}$ & & & & $\begin{array}{c}\text { Very } \\
\text { Comfortable }\end{array}$ & & & \\
\hline $\begin{array}{l}\text { 7. What areas of patient care are you most } \\
\text { confident in providing your ASD patients? }\end{array}$ & & & & & & & & \\
\hline $\begin{array}{l}\text { 8. Did you acquire any additional degrees, } \\
\text { licenses, or certifications related to the } \\
\text { quality of care you provide your patients } \\
\text { with ASD? }\end{array}$ & Yes & No & & & & & & \\
\hline \multicolumn{9}{|l|}{$\begin{array}{l}\text { 9. If your answer to Q8 is yes, what } \\
\text { additional degrees, licenses, or certifications } \\
\text { did you receive related to the quality of care } \\
\text { you provide your patients with ASD? Please } \\
\text { describe the nature of the additional training } \\
\text { you received (e.g., certificates obtained, } \\
\text { program attended, length of program). }\end{array}$} \\
\hline $\begin{array}{l}\text { 10. Does your employer offer training to } \\
\text { improve the quality of care you provide to } \\
\text { patients with ASD? }\end{array}$ & $\begin{array}{l}\text { Yes, I have } \\
\text { attended }\end{array}$ & $\begin{array}{l}\text { Yes, I have } \\
\text { not attended }\end{array}$ & No & I don't know & & & & \\
\hline \multicolumn{9}{|l|}{$\begin{array}{l}\text { 11. If your answer to Q10 is yes, what } \\
\text { additional training is offered by your } \\
\text { employer? Please describe the nature of the } \\
\text { additional training available (e.g., certificates } \\
\text { obtained, program attended, length of } \\
\text { program). }\end{array}$} \\
\hline $\begin{array}{l}\text { 12. If your answer to Q10 is yes, do you feel } \\
\text { the additional training you received from } \\
\text { your employer benefitted you and/or your } \\
\text { patients? }\end{array}$ & Yes & No & Unsure & & & & & \\
\hline $\begin{array}{l}\text { 13. If your answer to Q10 is yes, was the } \\
\text { additional training mandatory? }\end{array}$ & Yes & No & & & & & & \\
\hline $\begin{array}{l}\text { 14. Does your employer have a specific } \\
\text { protocol/guideline/instrument to aid in } \\
\text { communication with patients with ASD? }\end{array}$ & Yes & No & I don't know & & & & & \\
\hline \multicolumn{9}{|l|}{$\begin{array}{l}\text { 15. If your answer to Q14 is yes, please } \\
\text { describe the additional resources your } \\
\text { employer has available for you to use to aid } \\
\text { in communication with patients with ASD? }\end{array}$} \\
\hline $\begin{array}{l}\text { 16. If your answer to Q15 is yes, do you feel } \\
\text { these resources are helpful? }\end{array}$ & Yes & No & Unsure & & & & & \\
\hline \multicolumn{9}{|l|}{$\begin{array}{l}\text { 17. How can you improve the quality of care } \\
\text { you provide your patients with ASD? }\end{array}$} \\
\hline $\begin{array}{l}\text { 18. Do you feel that any of the following } \\
\text { would improve the quality of care you } \\
\text { provide to patients with ASD? }\end{array}$ & $\begin{array}{l}\text { Additional } \\
\text { training }\end{array}$ & $\begin{array}{l}\text { Continuing } \\
\text { education }\end{array}$ & $\begin{array}{l}\text { Communication } \\
\text { aids }\end{array}$ & Other: ........ & & & & \\
\hline
\end{tabular}




\section{RESULTS}

A total of 116 participants participated in the questionnaire over two-weeks period of data collection. Of the 116 participants, $73(62.9 \%)$ were pharmacists, $26(22.4 \%)$ were nurses, and $5(4.3 \%)$ were from medical sectors, and $12(10.3 \%)$ from other healthcare professionals such as occupational therapist, physical therapist, etc. (Figure 1a). The participants varied in years of experience (Figure 1b) from less than five years $(31 \%)$ to experience of 15 20 years $(8.6 \%)$. Those who have experience greater than 20 years were nearly similar to those with $10-15$ years of practice with $26.7 \%$ and $20.7 \%$, respectively. Figure 1c illustrates the practice setting of the healthcare provider participants. The majority of participants were in hospital-inpatient $(54.3 \%)$. Healthcare providers who work in community pharmacy and hospital-outpatient were nearly equal with $(14.6 \%)$ and $(13.7 \%)$, respectively. Other participants worked in outpatient clinic $(10.3 \%)$ and independent pharmacy $(2.5 \%)$. The majority of healthcare providers participated in the questionnaire were females with $69.8 \%$ compared to their male counterparts $29.3 \%$ (figure not shown).

Participants varied in their level of comfort when communicating with patients on the spectrum (Figure 2a). About 27\% of participants were either uncomfortable or totally uncomfortable communicating with adult patients on ASD directly, while $42.2 \%$ were not sure about their confidence level when dealing with patients on the spectrum. Only $10.3 \%$ were very comfortable communicating with patients on ASD, and $20.7 \%$ are somewhat comfortable. Figure $2 \mathrm{~b}$ shows the preparedness of questionnaire participants to provide quality care to patient on ASD. $33.6 \%$ of participants felt unprepared or totally unprepared to provide quality care to ASD patients while $42 \%$ were not sure about being prepared. Similar to their level of comfort (Figure $2 \mathrm{a}$ ), 25\% of participants felt very prepared or somewhat prepared to provide quality care to patients on the spectrum (Figure 2b).

Out of 116 questionnaire participants, only 4 (3.4\%) shared that they took additional training on their own to improve their ability to provide quality care, while the majority 112 (96.6\%) did not (Figure 3 ). When asked if the additional training was offered by the employer, $3(2.6 \%)$ said yes and they attended, and $4(3.4 \%)$ said although employer offers some training, they did not attend. The majority said their employers do not offer any training $78(67.2 \%)$, or they do not know if any training is being offered 31 $(26.7 \%)$. When asked if the training was mandatory, $1(14.3 \%)$ said yes, and $6(85.7 \%)$ said it was not mandatory. When asked if the training benefit them/their patients, $3(42.8 \%)$ said yes, and the majority $(57.2 \%)$ said no or unsure. When asked if there is any specific protocol/guideline in their workplace to facilitate their ability to provide quality care for patients on ASD, only $3(2.6 \%)$ said yes, and the majority $(97.4 \%)$ either said no or they are unaware of such protocols (please see Figure 3).

As suggestions to improve quality of care for patients on ASD, $98(86.7 \%)$ of questionnaire participants proposed continuing education courses/workshops could be beneficial, 89 (78.8\%) of participants believe additional training is needed, while 77 (68.1\%) think communication aids/tools would be helpful to improve their healthcare performance with ASD patients (Figure 2c). a

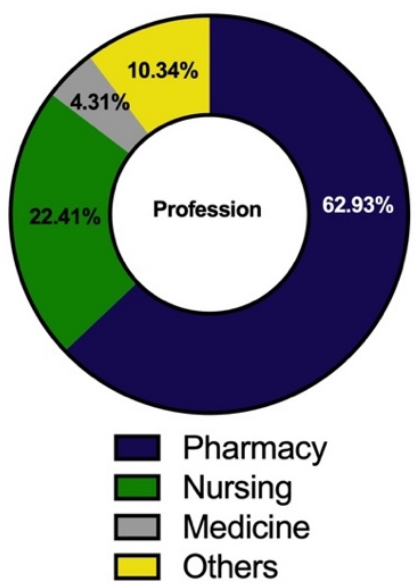

b

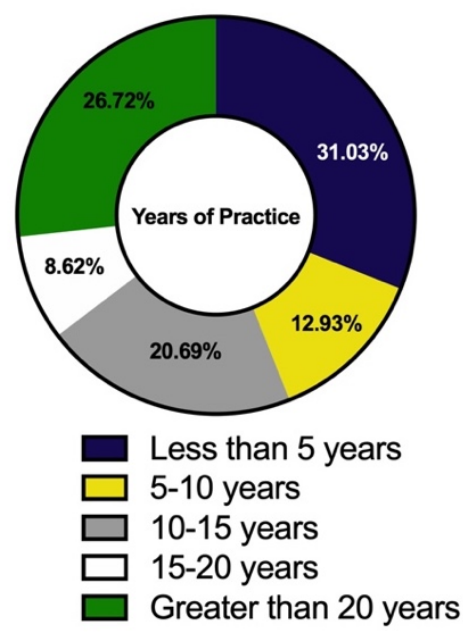

C

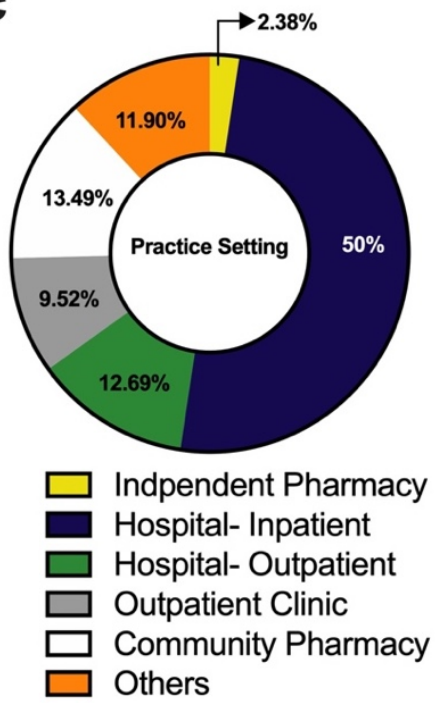

Figure 1. Questionnaire participants (a) Profession, (b) Years of practice, (c) Practice Setting. 
a

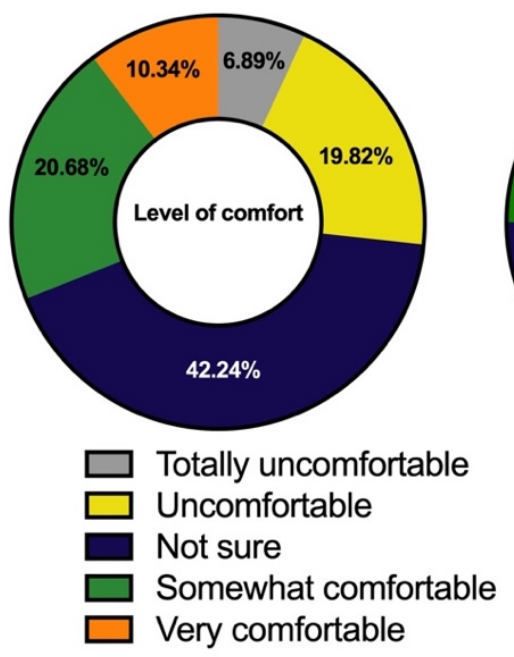

b

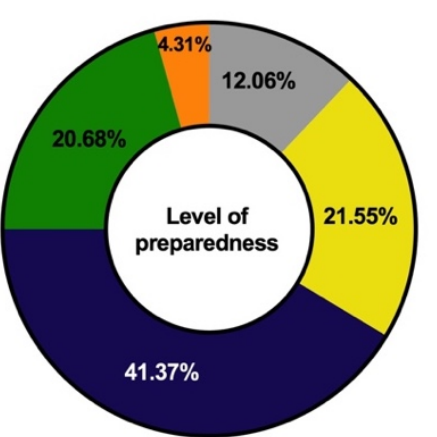

$\square$ Totally unprepared

$\square$ Unprepared

Not sure

$\square$ Somewhat prepared

$\square$ Very prepared
C

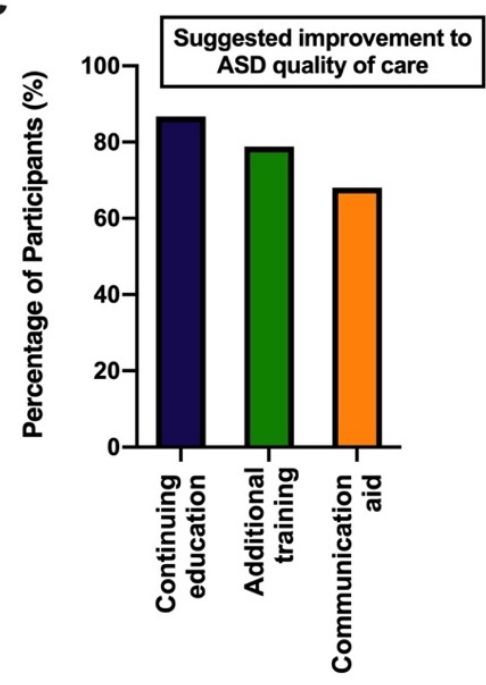

Figure 2. Questionnaire participants (a) Level of comfort (b) Level of preparedness to provide adult patients on ASD quality care, (c) Suggested improvement for the quality of care provided to adult patients on the spectrum

Figure 3 Questionnaire participants self-reported additional trainings/certificates to aid in providing quality care for adult patients with ASD.

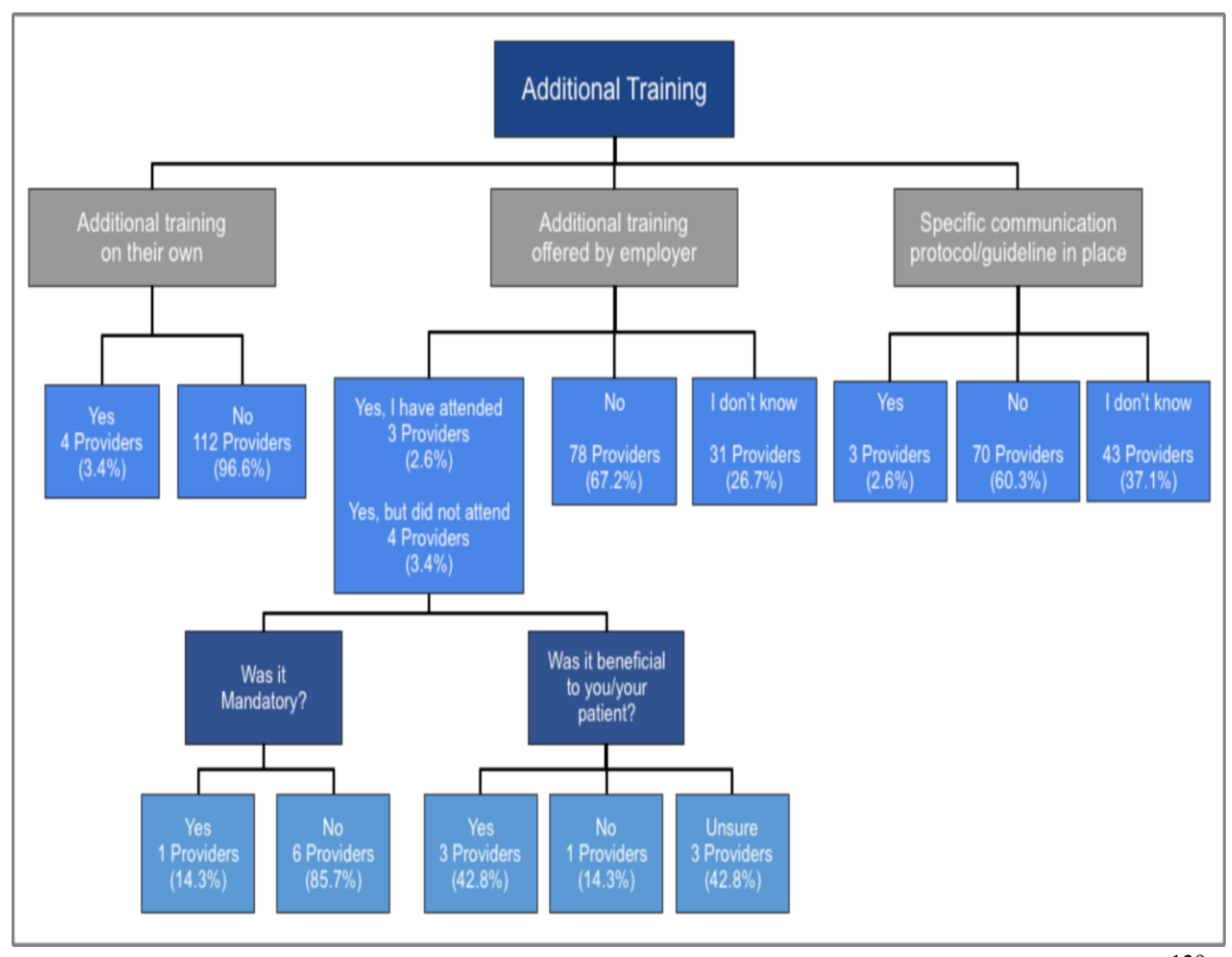




\section{DISCUSSION}

\subsection{Demographics}

Overall participation to our questionnaire was reasonable considering the time constraints of this project, as well as other limitations (see below). The questionnaire participants' years of experience spanned across 1-20 years, which makes the results herein a true reflection of the current healthcare providers' workforce regardless of the years of experience. Since the majority of our 116 questionnaire participants were pharmacists (63\%), and work in hospital-inpatient (54\%) (Figures 1b \& 1c), our discussion herein offers a good overall picture of how pharmacists perceive caring for adult patients with ASD.

\subsection{Comfort zones}

When asked how comfortable providers felt when communicating with patients on ASD, the results displayed a normal distribution curve where the majority of participants fall in the middle and tend to be unsure if they are comfortable or uncomfortable (42\%). Nicolaidis et al, 2020, reported that a very small percentage of healthcare providers who felt confident providing care to autistic adults patients (communication, physical procedure performance, accommodation needs identification among others) ${ }^{29}$. This is in line with other researchers reporting that pharmacist felt unconfident counseling parents about their ASD children's medication ${ }^{28}$. On the other side, there were $10 \%$ who reported feeling very comfortable in communicating with patients on the spectrum and $21 \%$ somewhat comfortable. It is worth to notice that those who felt comfortable referred that due to their personal experience as parents/caregivers/family members of someone on the spectrum (Figure $2 a$ ).

\subsection{Prepare to care}

Similar trends were observed when asked about the preparedness of health care providers to provide care to adult patients on the spectrum. The results displayed another normal distribution curve, where the majority of participants tend to be unsure if they are prepared or unprepared (41\%), and 33\% felt either totally unprepared (12\%) or unprepared $(21 \%)$. Only $4 \%$ reported feeling very prepared in providing quality care and $21 \%$ somewhat prepared (Figure 2b). When asked about the areas of patient care, providers felt most confident in providing, the responses varied significantly. Prescription monitoring, drug administration, preventative management, dispensing medications, and calming patients down to be able to give them their medications were among the answers given. Shawahna et al, 2017 commented that lack of necessary awareness among health care providers about ASD might be a contributing factor behind the lack of preparedness ${ }^{28}$. Zerbo et al, 2015 reported that there is more unmet health care needs and less satisfaction in adult patients with ASD compared to their neurotypical peers ${ }^{28}$.

\subsection{Communication spectrum}

A major barrier between patient and healthcare provider is the lack of communication which lead to incorrect diagnosis, inappropriate treatment or even person's avoidance of seeking health service ${ }^{30}$. In this context, verbal patient counseling was another area that most of the questionnaire participants were struggling with which reveal significant lack of awareness. However, few participants commented that the use of written communication rather than verbal can be effective. This takes us back to the ASD spectrum of cognitive and communication abilities for adults with ASD which can complicate identification and management of disease states unrelated to their disability ${ }^{16}$. Adults on the spectrum often need more time processing what they hear, they take words very literally, they do not read between the lines, or observe social cues. They usually learn best by visual cues, simple language, structured scripts, and reminders, usually prefer the ability to retrieve the information or learn it on their own pace 15,31. An assumption that a patient cannot understand healthcare information because they cannot speak frequently is incorrect. Similarly, an assumption that a patient with fluid speech or advanced vocabulary does not have significant communication difficulties is also inappropriate as concluded by Nicolaidis et al, $2016^{16}$.

\subsection{One size does not fit all}

Communication should be patient-specific, whenever possible. One size does not fit all, and this certainly adds a level of burden on the providers. ${ }^{32}$ Many questionnaire participants mentioned that they are more comfortable with pediatric patients because they can communicate easily with their parents/caregivers, but it is harder when it comes to adult patients. One participant said spending more time with their patients help him/her to understand them better and can tailor his/her communication strategy accordingly. Having said that, most participants commented that time is a big constrain when it comes to patient counseling. Providers should try to obtain as much information as possible about their patients' communication level and preferred communication mode prior to their visits, if and when possible. 


\subsection{Trainings/certificates}

Gap in knowledge and awareness is mainly attributed to lack of training and questionnaire participants were asked if they have received any additional training/certificate/license specific to ASD in their schooling or professional careers. Only $3 \%$ received training on their own and $6 \%$ were offered training from their employers. Even when the training was offered, more than half of them did not attend (Figure 3). Same low ratio (3\%) was for those who are aware of any communication protocol or guidelines in their own institutions. That leaves $97 \%$ who either do not know or unaware of any specific communication protocol or guidelines for communicating/counseling patients with ASD in their own institutions. It was reported that both primary and acute healthcare providers lack specialist training for communication with ASD patients and this may be related to time constraint and knowledge gap. ${ }^{30}$

The 3\% who received training on their own stated Autism Diagnostic Observation Schedule (ADOS) certification, critical issue in school health conference with ASD as a topic, and finally one participant mentioned that the Post-Graduate Year 2 (PGY2) in Psych and Board-Certified Psychiatric Pharmacist (BCPP) provided some additional education, though not all encompassing. None of these trainings are communication-based training programs. One participant revealed that he/she benefited from a statewide conference, although it was not mandatory by their employer. Few more mentioned some online mandatory training but were not as beneficial. Those who did not attend the additional training offered by their employer, generally speaking, reported that most of these training were online continuing education classes, and providers were unsure whether or not they would benefit from the training (Figure 3).

Evident by the poor satisfaction reported by the attendee of the current training sessions, it is quite apparent that there is a significant need to create more meaningful training events, and continuing educational sessions for health care providers to bring them up to speed when it comes to providing quality care for patients with ASD.

\subsection{Supply and Demand}

One of the common themes observed is that the need is more than the system supports. ${ }^{33}$ There is a less access to care of ASD specially outside metropolitan areas which leads to shortage of service supply, clinician exhaustion and long waiting list for treatment. Furthermore, additional burden (cost, time waste, travel) is added to families who do not have available care in their community. This decrease their willingness to seek care. ${ }^{34}$ Therefore, the urge for a collaborative care design for the treatment and management of ASD is emphasized ${ }^{33}$ The genuine desire of almost all providers to be better prepares to serve patients with ASD and provide optimal patient care was very encouraging. Questionnaire participants were given three choices, as well as, an open-ended option to add to at the end of the questionnaire on how to improve their quality of care they provide to adult patients with ASD. Imperative continuing education workshops specialized for patients with ASD was recommended by $87 \%$ of our participants, followed by additional training during their education/profession (79\%). Additionally, $68 \%$ believe they will benefit from exploring innovative communication aids, tools, or techniques that are effective with patients with ASD, and can be implemented in their workplace to improve the overall patient counseling experience (Figure 2c). Some of the meaningful adds-on suggestions were; build team approach, increase staff awareness of the healthcare barriers, provide sensory friendly environment, promote patience and compassion, support from a specialist in the field, provide less distracting area for patient counseling, process prescription faster to avoid unstructured waiting times, and book longer visits with the patient.

We believe that pharmacists, especially in community settings, should be among the forefront of health care providers who would benefit significantly from gathering this communication level information, especially for recurrent patients on the spectrum. Information from this study encouraged our research team to design an innovative augmentative communication aid in form of a mobile app to be used by the patient and the healthcare provider for transfer/exchange/retrieval of information. The communication aid will be piloted in the next phase of this research.

\section{LIMITATIONS}

Some limitations to our study, that we are aware of, includes time constraints of data collection since this was conducted through a 6-week Advanced Pharmacy Practice Rotation; the limited access to healthcare providers in larger healthcare institutes who opted not to respond to our communication; and lastly cyber security prevented some organizations who opted to respond back to share the questionnaire to their providers.

\section{CONCLUSIONS}

Our study concludes that there is an urgent need to provide better, more meaningful ASD training and education programs for healthcare providers in 
general, and pharmacists in particular, geared towards communication. Because of the wide spectrum of ASD, and the wide range of individual communication differences, communication should always be patient-specific to undermine stigma and prevent any prejudice bias or disparities.

Providers shared their desire to bridge the communication gap and willingness to explore the use of innovative communication tools, techniques, strategies that can improve their caring for adult patients on the ASD. Information from this study encouraged our research team to design an innovative augmentative communication aid in form of a mobile app to be used by the patient and the health care provider for transfer/exchange/retrieval of information. The communication aid will be piloted in the next phase of this research.

Funding: This research did not receive any grant from funding agencies in the public, commercial, or not-for-profit sectors.

Conflicts of interest: Authors declare no competing financial interests exist

\section{Acknowledgment: NA}

Ethical Statement: The research has been approved by the Institutional Review Board (IRB) at the University of Saint Joseph IRB Protocol Number: 18-0054.

Author Contribution: Dr. Ola Ghoneim was the main preceptor who supervised the two pharmacy students (Luis Manuel Lavoura and Noelle Bianculli) under their Advanced Pharmacy Practice Experience at the University of Saint Joseph. She orchestrated all aspects of the project and wrote the main sections of the manuscript. Dr. Heba Eassa helped significantly in the data collection and data analysis and the write up of this manuscript. Luis Manuel Lavoura and Noelle Bianculli conducted the literature search needed to get the appropriate referencing. They helped with the creation of the questionnaire questions, getting a list of healthcare provider email distribution list, distributing the questionnaire, and following up as the main communicators of the project. They were also trained and certified by the $\mathrm{NIH}$ during this project. Dr. Charlotte Mann was the main player in the creation of the questionnaire questions, using the appropriate language to all questions to avoid unintended bias, and the submission of the project to the Institutional Review Board (IRB) at the University of Saint Joseph.
The manuscript has been submitted solely to this journal, and is not published, in press, or submitted elsewhere.

\section{REFERENCES}

1. Healthy People 2020 Midcourse Review. National Center for Health Statistics 2018 [Available

from:https://www.cdc.gov/nchs/healthy_pe ople/hp2020/hp2020_midcourse_review.ht m.

2. Axmon A, Sandberg M, Ahlström G, Midlöv PJBP, Toxicology. Prescription of potentially inappropriate medications among older people with intellectual disability: a register study. 2017;18(1):110 .

3. Centers for Disease Control and Prevention. Data \& Statistics on Autism Spectrum Disorder. 2021 [updated December 2, 2021; cited December 5, 2021]. Available from: https://www.cdc.gov/ncbddd/autism/data.h tml.

4. Autism Speaks. Autism Facts and Figures 2018 [October 13th, 2019]. Available from: https://www.autismspeaks.org/autismfacts-and-figures.

5. Robison JE. Autism prevalence and outcomes in older adults. 2019;12(3):370-4.

6. Cakir J, Frye RE, Walker SJJRiASD. The lifetime social cost of autism: 1990-2029. 2020;72:101502.

7. Kheir N, Ghoneim O, Sandridge AL, AlIsmail M, Hayder S, Al-Rawi FJA. Quality of life of caregivers of children with autism in Qatar. 2012;16(3):293-8.

8. Kheir NM, Ghoneim OM, Sandridge AL, Hayder SA, Al-Ismail MS, Al-Rawi FJBrn. Concerns and considerations among caregivers of a child with autism in Qatar. 2012;5(1):1-7.

9. Brown AB, Elder JHJPN. Communication in autism spectrum disorder: a guide for pediatric nurses. 2014;40(5):219.

10. Tager-Flusberg $\mathrm{H}$, Paul $\mathrm{R}$, Lord C. Language and communication in autism. 2005. 
11. Edition FJAPA. Diagnostic and statistical manual of mental disorders. 2013;21.

12. Brignell A, Chenausky KV, Song H, Zhu J, Suo C, Morgan ATJCDoSR. Communication interventions for autism spectrum disorder in minimally verbal children. 2018(11).

13. Schriber RA, Robins RW, Solomon MJJop, psychology s. Personality and self-insight in individuals with autism spectrum disorder. 2014;106(1):112.

14. Cummins C, Pellicano E, Crane L. Autistic adults' views of their communication skills and needs. International journal of language \& communication disorders. 2020;55(5):678-89.

15. Lartseva A, Dijkstra T, Buitelaar JKJFihn. Emotional language processing in autism spectrum disorders: a systematic review. 2015;8:991.

16. Raymaker DM, McDonald KE, Ashkenazy E, Gerrity M, Baggs AM, Kripke C, et al. Barriers to healthcare: Instrument development and comparison between autistic adults and adults with and without other disabilities. Autism : the international journal of research and practice. 2016;21(8):972-84.

17. Bolick TJTiLD. "It takes a community": Social capital, autism spectrum disorders, and the real world. 2008;28(4):375-87.

18. Raymaker DM, McDonald KE, Ashkenazy E, Gerrity M, Baggs AM, Kripke C, et al. Barriers to healthcare: Instrument development and comparison between autistic adults and adults with and without other disabilities. 2017;21(8):972-84.

19. Buie T, Campbell DB, Fuchs GJ, 3rd, Furuta GT, Levy J, Vandewater J, et al. Evaluation, diagnosis, and treatment of gastrointestinal disorders in individuals with ASDs: a consensus report. Pediatrics. 2010;125 Suppl 1:S1-18.

20. Rada RE. Treatment needs and adverse events related to dental treatment under general anesthesia for individuals with autism. Intellectual and developmental disabilities. 2013;51(4):246-52.
21. Preißmann C. Autism and healthcare. Advances in Autism. 2017;3(3):115-24.

22. Tregnago MK, Cheak-Zamora NC. Systematic review of disparities in health care for individuals with autism spectrum disorders in the United States. Research in Autism Spectrum Disorders. 2012;6(3):1023-31.

23. Hand BN, Angell AM, Harris L, Carpenter LAJA. Prevalence of physical and mental health conditions in Medicare-enrolled, autistic older adults. 2020;24(3):755-64.

24. Nicolaidis C, Raymaker D, McDonald K, Dern S, Ashkenazy E, Boisclair C, et al. Collaboration strategies in nontraditional community-based participatory research partnerships: Lessons from an academiccommunity partnership with autistic selfadvocates. 2011;5(2):143.

25. Nicolaidis C, Raymaker D, McDonald K, Dern S, Boisclair WC, Ashkenazy E, et al. Comparison of healthcare experiences in autistic and non-autistic adults: a crosssectional online survey facilitated by an academic-community partnership. Journal of general internal medicine. 2013;28(6):761-9.

26. Saqr Y, Braun E, Porter K, Barnette D, Hanks C. Addressing medical needs of adolescents and adults with autism spectrum disorders in a primary care setting. Autism : the international journal of research and practice. 2018;22(1):51-61.

27. Crossley KJPT. Public perceives pharmacists as some of the most trusted professionals. 2019;18.

28. Ensing HT, Vervloet M, van Dooren AA, Bouvy ML, Koster ES. Patient-pharmacist communication during a post-discharge pharmacist home visit. Int J Clin Pharm. 2018;40(3):712-20.

29. Nicolaidis C, Schnider G, Lee J, Raymaker DM, Kapp SK, Croen LA, et al. Development and psychometric testing of the AASPIRE Adult Autism Healthcare Provider Self-Efficacy Scale. Autism. 2020;25(3):767-73. 
30. Doherty AJ, Atherton $\mathrm{H}$, Boland $\mathrm{P}$, Hastings R, Hives L, Hood K, et al. Barriers and facilitators to primary health care for people with intellectual disabilities and/or autism: an integrative review. 2020;4(3).

31. Wilson A, Bishop DJRSos. Judging meaning: A domain-level difference between autistic and non-autistic adults. 2020;7(11):200845.

32. Iannuzzi D, Kopecky K, Broder-Fingert S, Connors SLJH, work s. Addressing the needs of individuals with autism: role of hospital-based social workers in implementation of a patient-centered care plan. 2015;40(3):245-8.

33. Pope K, Doll J, Kyvelidou A, Stessman H, Nelson K, Jordan LJJoIE, et al. Clinician, caregiver and patient perspectives of the continuum of care for autism. 2020;19:100335.

34. Malik-Soni N, Shaker A, Luck H, Mullin AE, Wiley RE, Lewis MS, et al. Tackling healthcare access barriers for individuals with autism from diagnosis to adulthood. 2021:1-8. 